\title{
Contrasting effects of specialist and generalist herbivores on resistance evolution in invasive plants
}

\author{
Zhijie Zhang, ${ }^{1,2}$ Xiaoyun Pan, ${ }^{1,5}$ Dana Blumenthal, ${ }^{3}$ Mark van Kleunen, ${ }^{4,2}$ Mu Liu, ${ }^{1}$ And Bo Li ${ }^{1}$ \\ ${ }^{1}$ Ministry of Education Key Laboratory for Biodiversity Science \& Ecological Engineering, Institute of Biodiversity Science, \\ Fudan University, Shanghai 200438 China \\ ${ }^{2}$ Ecology, Department of Biology, University of Konstanz, Konstanz 78464 Germany \\ ${ }^{3}$ USDA-ARS Rangeland Resource Research Unit, Fort Collins, Colorado 80526 USA \\ ${ }^{4}$ Zhejiang Provincial Key Laboratory of Plant Evolutionary Ecology and Conservation, Taizhou University, Taizhou 318000 China
}

\begin{abstract}
Invasive alien plants are likely to be released from specialist herbivores and at the same time encounter biotic resistance from resident generalist herbivores in their new ranges. The Shifting Defense hypothesis predicts that this will result in evolution of decreased defense against specialist herbivores and increased defense against generalist herbivores. To test this, we performed a comprehensive meta-analysis of $61 \mathrm{common}$ garden studies that provide data on resistance and/or tolerance for both introduced and native populations of 32 invasive plant species. We demonstrate that introduced populations, relative to native populations, decreased their resistance against specialists, and increased their resistance against generalists. These differences were significant when resistance was measured in terms of damage caused by the herbivore, but not in terms of performance of the herbivore. Furthermore, we found the first evidence that the magnitude of resistance differences between introduced and native populations depended significantly on herbivore origin (i.e., whether the test herbivore was collected from the native or non-native range of the invasive plant). Finally, tolerance to generalists was found to be higher in introduced populations, while neither tolerance to specialists nor that to simulated herbivory differed between introduced and native plant populations. We conclude that enemy release from specialist herbivores and biotic resistance from generalist herbivores have contrasting effects on resistance evolution in invasive plants. Our results thus provide strong support for the Shifting Defense hypothesis.
\end{abstract}

Key words: biotic resistance; enemy release; evolution of herbivore defense; invasive plant species; meta-analysis; resistance traits and effects; specialist-generalist paradigm; tolerance.

\section{INTRODUCTION}

Concern over the growing prevalence and impacts of plant invasions has stimulated interest in determinants of invasiveness (Vilà et al. 2011, van Kleunen et al. 2015, Seebens et al. 2017). An intuitive and long-standing hypothesis is that many invasive plants in their non-native ranges have escaped suppression by co-evolved enemies, primarily specialists (Keane and Crawley 2002, Liu and Stiling 2006). Building on this Enemy Release hypothesis (ERH), another hypothesis, the Evolution of Increased Competitive Ability (EICA) hypothesis, poses that selection in the non-native range would favor increased investment in growth or competitive ability at the cost of herbivore defense (Blossey and Nötzold 1995). While most studies have attributed the evolution of herbivore defense to enemy release, invasive plants are nevertheless likely to encounter potentially strong biotic resistance from an array of generalist herbivores in their non-native ranges (i.e., a component of the Biotic Resistance hypothesis, BRH; Elton 1958, Maron and Vilà 2001, Levine et al. 2004, Parker et al. 2006, Morrison and Hay 2011). Consequently, selection in invasive plants may not only lead to evolution of reduced defenses against specialist herbivores but also to evolution of higher levels of defense against generalist herbivores (Shifting Defense hypothesis, SDH; Müller-Schärer et al. 2004, Joshi and Vrieling 2005). Despite strong interest in the evolution of invasive plants (e.g., Colautti and Barrett 2013, Oduor et al. 2016), we still lack a comprehensive assessment of the evolution of herbivore defense in invasive plants.

A fact that complicates the study of herbivore-defense evolution is that plants can have a wide variety of defense strategies, which vary both in their costs to the plant and their effects on different herbivores (Koricheva 2002, Kempel et al. 2011). Resistance and tolerance are widely acknowledged as two major components of herbivore defense (Núñez-Farfán et al. 2007, Carmona and Fornoni 2013). Resistance by chemical and physical defenses is considered to avoid or reduce damage by herbivores and reduce performance of herbivores, and tolerance is considered to reduce the impact of herbivory on the plant. Predictions of how defense against herbivores will evolve following introduction hinge on both the presence and nature of enemies in the non-native ranges and the relative costs of the different types of defenses (Fig. 1).

Resistance is assumed to be relatively costly (Stamp 2003), and has therefore been the primary focus of studies testing the Shifting Defense hypothesis. Although the costs of different resistance mechanisms may depend on resource availability (Coley et al. 1985), it is thought that physical defenses (e.g., trichomes) and high-concentration digestibility-reducers (e.g., tannins) are particularly expensive (Feeny 1975, Müller-Schärer et al. 2004). These two types of defenses are 
(a) Defense against specialists

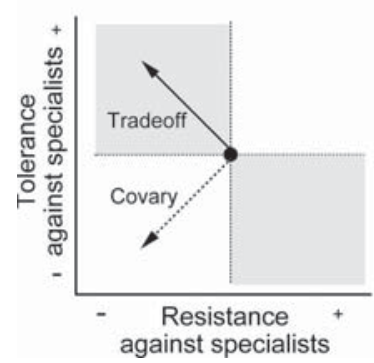

(b) Defense against generalists

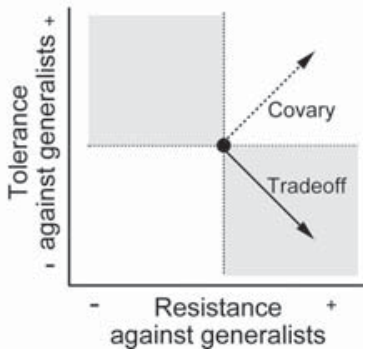

FIG. 1. Predictions for how resistance and tolerance may evolve following invasion. (a) Resistance against specialist enemies is expected to decline due to release from specialist enemies, as predicted by the Shifting Defense hypothesis (SDH), while tolerance might increase due to tradeoffs among defense strategies or decrease due to positive covariance. (b) Conversely, resistance against generalists is predicted to increase due to biotic resistance, as predicted by the Shifting Defense hypothesis (SDH). Tolerance might decrease due to tradeoffs, or increase due to positive covariance. Grey and white zones represent predictions of defense evolution due to tradeoff or positive covariance, respectively. Solid and dotted arrows represent specific predictions of defense evolution following introduction due to tradeoff or positive covariance.

hypothesized to be effective against specialist as well as generalist herbivores (Feeny 1975, Müller-Schärer et al. 2004). Another mechanism of resistance is provided by toxins (e.g., alkaloids and glucosinolates), which are already effective in low concentrations and therefore less expensive. Toxins are hypothesized to be primarily effective against, and therefore selected for by, generalists (Müller-Schärer et al. 2004). On the other hand, toxins might be selected against, because they might be tolerated by or even attract adapted specialists (van der Meijden 1996). The Shifting Defense hypothesis predicts that loss of specialists in the non-native range will relax this specialist-generalist dilemma and lead to evolution of higher levels of resistance traits against generalists (i.e., toxins) and lower levels of resistance traits against specialists (i.e., digestibility-reducers and physical defenses) in invasive plants (Müller-Schärer et al. 2004, Joshi and Vrieling 2005) (Fig. 1).

A previous meta-analysis found that toxins of invasive plants indeed increased significantly in the non-native ranges, whereas digestibility reducers and physical defenses did not change significantly (Doorduin and Vrieling 2011). Although these findings partly support the Shifting Defense hypothesis, criticisms have been levied against directly categorizing resistance traits as those against specialists or generalists. For example, toxins were reported to be both effective against specialist and generalist herbivores (Smilanich et al. 2016), and some digestibility-reducers could act as feeding stimulants for specialists (Schultz 1989). Consequently, whether evolution of resistance traits could translate into evolution of resistance effects (i.e., damage by and performance of herbivores) is unknown. Several experimental studies that evaluated damage by and/or performance of herbivores in native and introduced populations of invasive species found equivocal results (Wolfe et al. 2004, Joshi and Vrieling 2005, Ridenour et al. 2008, Pan et al. 2012). Therefore, it is important to consider both resistance traits and resistance effects when testing the Shifting Defense hypothesis.

Tolerance to herbivory, another defense strategy that is considered to be adaptive and costly (Stowe et al. 2000, Núñez-Farfán et al. 2007), has received much less attention than resistance. The evolution of tolerance to herbivory in invasive species has been subjected to conflicting predictions (Fig. 1), and the results are mixed (van Kleunen and Schmid 2003, Bossdorf et al. 2005, Huang et al. 2010, Zhang et al. 2015). Classical theory predicts tradeoffs between resistance and tolerance because both are costly (Van der Meijden et al. 1988). Following this logic, resistance and tolerance of invasive plants should evolve in opposite directions (Fig. 1a, b solid arrows). Nonetheless, little evidence has been found for the proposed tradeoff (Leimu and Koricheva 2006, Carmona and Fornoni 2013, Mesa et al. 2017). Furthermore, theoretical research showed that resistance and tolerance could positively covary if herbivore pressure is high (Fornoni et al. 2004). Following this logic, resistance and tolerance will evolve in the same direction (Fig. 1a, b dotted arrows).

An important source of variation in the results of empirical studies on defense evolution in invasive plants might be the origin of the herbivores used to damage the plants when tolerance or the resistance effects are assessed. When invasive plants are locally adapted (Lee 2002, Colautti et al. 2009), introduced populations of invasive plants are likely to have increased defense against herbivores co-occurring with them (i.e., herbivores from non-native ranges of the invasive plants), relative to native populations, but decreased defense against herbivores no longer co-occurring with them (i.e., herbivores from native ranges of the invasive plants). Therefore, it might be important to consider the origin of the bioassay herbivore when assessing defense evolution in invasive plants. To the best of our knowledge, however, the importance of herbivore origin has rarely been addressed (but see Schaffner et al. 2011, Bukovinszky et al. 2014).

Most comparative experiments testing these questions included only one plant and/or herbivore species and a single defense strategy. Narrative literature reviews of such case studies employed a vote-counting approach, which do not allow for rigorous hypothesis testing (Borenstein et al. 2009). Therefore, we conducted a meta-analysis that allowed us to combine information from case studies to provide more general insights and to examine the sources of variation of previous empirical studies. Three previous meta-analyses have also addressed evolution of defenses in invasive plants. Colautti et al. (2009) and Felker-Quinn et al. (2013) reviewed studies concerning rapid evolution in invasive plants in general and found no support for evolution in herbivore resistance. Doorduin and Vrieling (2011) focused on studies testing evolution of resistance traits in invasive plants and found that toxins had increased in the introduced populations. Still missing, however, is a comprehensive meta-analysis that synthesizes the ever-growing literature on invasive plant defense evolution, and differentiates between multiple defense strategies (resistance traits, resistance effects and tolerance), multiple herbivore types (specialists and generalists) and herbivore origin (native and non-native range).

To address these knowledge gaps, we considerably expanded the datasets used in the previous meta-analyses. The first objective of our meta-analysis was to test whether introduced populations of invasive plants have evolved reduced defenses (both resistance and tolerance) against 
specialist herbivores, and increased defenses against generalist herbivores, when compared with native populations. Our second objective was to test whether the detection of defense evolution in invasive plants depends on herbivore origin. Particularly, we expected to find an increased defense in introduced populations when using test herbivores collected from the non-native range of the invasive plant, and to find a decreased defense when using a test herbivore from the native range.

\section{Methods}

\section{Data selection}

We conducted a literature search in Web of Science for the terms ("defense OR resistance OR tolerance" "plant" "herbivor* OR insect OR enemy" "invasi* OR introduce* OR exotic OR alien"), and searched all publications up until December 2015. Additional papers were identified by examining the references of previous reviews and meta-analyses on the role of evolution in plant invasions (Bossdorf et al. 2005, Colautti et al. 2009, Doorduin and Vrieling 2011, Felker-Quinn et al. 2013). Studies were individually assessed and retained if all of the following four criteria were met. (1) The study focused on defense-related traits of both introduced and native populations of invasive plant species, and was conducted in a common environment. (2) The nonnative and native ranges were each represented by at least two geographically distinct populations. This criterion was used because evolution of invasive plant defense emphasizes the difference between ranges, each of which consists of different populations experiencing various biotic and abiotic conditions. Studies that used only one population from each range could only test the difference between the two populations, which may not be representative for the difference between ranges. (3) The study provided information on whether the insect herbivore used was a specialist or a generalist. (4) Data could be obtained for the mean values and variances (standard deviations or standard errors) of defense-related traits and numbers of populations used for both the native and non-native ranges.

\section{The dataset}

We identified 60 published papers that met our criteria (Appendix S1), and also included data from one of our own studies, which was published later (Liu et al. 2017). From these sources, we extracted data for 32 plant species and 272 individual defense-related trait measurements. We categorized the traits into: (1) traits providing resistance, including concentrations of physical defenses, digestibility-reducers, and toxins; (2) traits that quantify resistance effects, including damage by and performance of herbivores; (3) tolerance (in terms of biomass production, growth rate or reproduction of plants). We distinguished between defenses against generalist and specialist herbivores. For tolerance, we also included tolerance to simulated herbivory as a separate category (Table 1).

We extracted the means and variances (standard deviations or standard errors) of each defense-related variable, and the sample sizes for introduced populations and native populations. These data were extracted directly from the text and tables or from graphs using the GetaData software (http://ge tdata-graph-digitizer.com). As some publications provided data on multiple plant or herbivore species, for multiple sites, census dates or for multiple defense-related traits, we adopted the following criteria to select the case studies (units) for analysis. If a publication reported data for more than one invasive plant species, herbivore species or study site, each of them was considered to be an independent case study. If the same study reported data for multiple points in time, only the final data point (longest duration of the study) was included. These criteria match the ones of other meta-analyses (van Kleunen et al. 2010, Sorte et al. 2013). Some studies reported more than one trait per defense category for a species. To avoid pseudo-replication, we pooled effect sizes and variances for each defense category per invasive plant species in a study by doing a separate fixed effect meta-analysis (which assumes that there is only one true effect size per study) on all traits and environments of the respective defense category. The estimated pooled mean effect size and the mean variance were used in the final data set.

In order to identify the origins of herbivores used in each case study, we extracted data on whether the herbivore had been collected from the native range of the invasive plant, and thus was likely to co-occur with native populations of the plant, or from the non-native range of the invasive plant, and thus was likely to co-occur with introduced populations. A few herbivore species in our dataset (e.g., Aphis nerii in Bukovinszky et al. 2014; Trichoplusia ni in Leger and Forister 2005 and Ridenour et al. 2008) were collected from both the native and the non-native range of the invasive plant. In these cases, we considered the herbivore populations collected from the different ranges as different herbivores, because they differ in their ecological and evolutionary history with the native and introduced populations of the invasive plant.

TABLE 1. Categories and examples of defense-related traits as obtained from the publications included in the meta-analysis.

\begin{tabular}{|c|c|c|c|}
\hline \multicolumn{3}{|c|}{ Defense category } & \multirow[b]{2}{*}{ Traits } \\
\hline Type of defense & Measure & Herbivore & \\
\hline \multirow[t]{3}{*}{ Resistance traits } & Physical defense & - & Trichomes, latex, cellulose \\
\hline & Digestibility-reducer & - & Tannins, lignin \\
\hline & Toxin & - & Pyrrolizidine alkaloids, glucosinolates etc. \\
\hline \multirow[t]{2}{*}{ Resistance effects } & Damage by herbivore & S, G & Number, percent, area or biomass of damaged leaves, biomass of damaged fruits \\
\hline & Performance of herbivore & $\mathrm{S}, \mathrm{G}$ & Survival, biomass, growth rate, development time, population size, oviposition \\
\hline Tolerance & - & $\mathrm{S}, \mathrm{G}, \mathrm{SH}$ & Biomass, growth rate, reproduction \\
\hline
\end{tabular}

Notes: S, specialist herbivores; G, generalist herbivores; SH, simulated herbivory. 


\section{Calculation of effect sizes}

We used the log response ratio as effect size (Borenstein et al. 2009). However, as this metric can be considerably biased when the studies have small sample sizes, we used a bias-corrected effect size (Lajeunesse 2015). For resistance traits (e.g., lignin content) and effects (damage by and performance of herbivores), we calculated the difference between introduced and native populations for each species in a study as:

$$
\begin{aligned}
\operatorname{Ln} R= & \ln \left(\bar{X}_{\text {introduced }}\right)-\ln \left(\bar{X}_{\text {native }}\right) \\
& +\frac{1}{2}\left[\frac{S_{\text {introduced }}^{2}}{n_{\text {introduced }} \bar{X}_{\text {introduced }}^{2}}-\frac{S_{\text {naitve }}^{2}}{n_{\text {native }} \bar{X}_{\text {native }}^{2}}\right]
\end{aligned}
$$

where $\bar{X}_{\text {introduced }}$ and $\bar{X}_{\text {native }}$ are the means of a trait of the introduced populations and the native populations, respectively, and where $S$ and $n$ are the standard deviations and sample sizes, respectively. When a low value of a trait indicates high resistance (e.g., small damage by herbivore indicates high resistance effect), we changed the sign of the effect size to make sure that positive $\operatorname{Ln} R$ values of resistance effect sizes indicate that introduced populations had higher resistance effect sizes than the native ones, and negative $\operatorname{Ln} R$ values indicate the opposite. Effect size variance (v) was estimated as:

$$
\begin{aligned}
v= & \frac{S_{\text {introduced }}^{2}}{n_{\text {introduced }} \bar{X}_{\text {introduced }}^{2}}+\frac{S_{\text {naitve }}^{2}}{n_{\text {native }} \bar{X}_{\text {native }}^{2}} \\
& +\frac{1}{2}\left[\frac{S_{\text {introduced }}^{4}}{n_{\text {introduced }}^{2} \bar{X}_{\text {introduced }}^{4}}+\frac{S_{\text {naitve }}^{4}}{n_{\text {native }}^{2} \bar{X}_{\text {native }}^{4}}\right]
\end{aligned}
$$

For tolerance, which in contrast to resistance is based on the comparison in performance between a control plant and a damaged plant, we first calculated an effect size (log response ratio, $\operatorname{Ln} R$ ) and variance for the performance measure (biomass, growth rate or reproduction) and herbivore treatment for introduced and native populations of each invasive plant species separately as:

$$
\begin{aligned}
\operatorname{Ln} R_{i} & =\ln \left(\bar{X}_{\mathrm{t}}\right)-\ln \left(\bar{X}_{\mathrm{c}}\right)+\frac{1}{2}\left[\frac{S_{\mathrm{t}}^{2}}{n_{\mathrm{t}} \bar{X}_{\mathrm{t}}^{2}}-\frac{S_{\mathrm{c}}^{2}}{n_{\mathrm{c}} \bar{X}_{\mathrm{c}}^{2}}\right] \\
v_{i} & =\frac{S_{\mathrm{t}}^{2}}{n_{\mathrm{t}} \bar{X}_{\mathrm{t}}^{2}}+\frac{S_{\mathrm{c}}^{2}}{n_{\mathrm{c}} \bar{X}_{\mathrm{c}}^{2}}+\frac{1}{2}\left[\frac{S_{\mathrm{t}}^{4}}{n_{\mathrm{t}}^{2} \bar{X}_{\mathrm{t}}^{4}}+\frac{S_{\mathrm{c}}^{4}}{n_{\mathrm{c}}^{2} \bar{X}_{\mathrm{c}}^{4}}\right]
\end{aligned}
$$

Here, $i$ refers to either introduced or invasive populations, $\bar{X}_{\mathrm{t}}$ and $\bar{X}_{\mathrm{c}}$ are the means of a performance variable in the herbivory damage treatment (t) and the control (c), and $S$ and $n$ are the corresponding standard deviations and sample sizes, respectively.

We used the value of $\operatorname{Ln} R$ as our measure of tolerance for the introduced and native populations of a given species. Then to assess whether tolerance differed between introduced and native populations, we calculated an effect size for the difference in tolerance between the groups of introduced and native populations of a species:

$$
\mathrm{Ln} R_{\text {difference }}=\operatorname{Ln} R_{\text {introduced }}-\operatorname{Ln} R_{\text {native }}
$$

Positive $\operatorname{Ln} R_{\text {difference }}$ values indicate that tolerance is higher in the introduced populations than the native ones, and negative values indicate the opposite. Ideally, the effectsize variance would be calculated as:

$$
v_{\text {difference }}=v_{\text {introduced }}+v_{\text {native }}-2 r \sqrt{v_{\text {introduced }}} \sqrt{v_{\text {native }}}
$$

where $r$ is the correlation coefficient describing the extent to which $\operatorname{Ln} R_{\text {invasive }}$ and $\operatorname{Ln} R_{\text {native }}$ co-vary (Borenstein et al. 2009). However, since the correlation could not be extracted from each study, the variance for each effect size was instead estimated as:

$$
v_{\text {difference }}=\frac{v_{\text {introduced }}+v_{\text {native }}}{2}
$$

As this might underestimate the true variance (Borenstein et al. 2009), we also estimated it as the sum of $v_{\text {introduced }}$ and $v_{\text {native, }}$ which might overestimate the variance. The results were quite similar, indicating that they are robust (Appendix S2: Table S1).

\section{Data-analysis}

After calculation of effect sizes for each study, we first used random-effect models to calculate the mean effect size of each defense category (metafor package for R 3.0.3; Viechtbauer 2010, R Development Core Team 2013). This implies that we assumed that differences among studies are not only due to sampling error but also due to true random variation, as is the rule for ecological data (Borenstein et al. 2009). Defense evolution in invasive plant species (i.e., a difference in defenses between native and introduced populations) is considered to be statistically significant if the $95 \%$ confidence interval of the mean effect size does not include zero. A votecounting approach was also employed as a comparison with meta-analysis, and the results are in line with the results of this meta-analysis (Appendix S3: Table S1).

Next, we used mixed-effects multivariate models to test whether defense evolution was dependent on herbivore origin, plant life form or the use of the herbivore as biological control agent. Herbivore origin was included to test for potential adaptation of the invasive plant to the herbivores in the native or non-native ranges. We included life form of the invasive plant, because long-lived and conspicuous woody species are expected to show higher defense levels than shortlived and inconspicuous herbaceous species (Feeny 1976, Cates and Rhoades 1977). We included herbivore use as biological control agent in the model, because several studies tested defense against specialist herbivores with biological control agents, which are carefully selected to have strongly negative effect on invasive plants (Seastedt 2015).

We used likelihood ratio tests to determine the significance of effects of the moderator variables (i.e., herbivore origin, life form, use as biological control agent) on defense evolution in invasive plants. This was done by comparing a full model containing all the moderators with a reduced model where a moderator of interest was removed. Caution 
should be exercised in interpreting the results of life form, given that there are only three woody species included in our dataset (Appendix S4: Table S1; Fig. S2).

The appropriate way to measure tolerance has been debated for years (Tiffin and Inouye 2000, Lehtilä 2003, de Jong and Lin 2017). Firstly, tolerance estimates may change, depending on the performance measure assessed. Ideally, tolerance should be measured in terms of fitness (e.g., survival and reproduction), but most studies measured plant growth (e.g., biomass and growth rate) instead, for practical reasons. Secondly, tolerance estimates may be dependent on whether one uses the same or different genetic material in control and herbivory treatments (Stowe et al. 2000, Lehtilä 2003). Thirdly, estimates of tolerance may depend on whether damage level is consistent within the damage treatments, because different damage levels might stimulate different regrowth rates (Tiffin and Inouye 2000). Therefore, we used likelihood ratio tests as described above to test the effects of study design (i.e.,, whether tolerance is measured in terms of reproduction or growth, whether genetic background is controlled or not, and whether damage level is controlled or not) on the observed difference in tolerance between introduced and native populations. We did not distinguish between tolerance to specialist, generalist and simulated herbivory when testing these three factors, since only a few studies in our dataset used the same genetic material across herbivory treatments (Joshi and Vrieling 2005, Meyer and Hull-Sanders 2008, Zhang et al. 2015), and studies that controlled the damage level usually applied simulated herbivory. The three factors tested in our study had no significant effects on mean effect sizes of differences in tolerance between introduced and native populations (Appendix S5: Table S1).

We also compared our dataset of plant resistance traits with the one of Doorduin and Vrieling (2011) using cumulative meta-analysis to assess whether and how the mean effect sizes of physical defenses, digestibility-reducers, and toxins changed with $6 \mathrm{yr}$ of additional data. Cumulative meta-analysis is a series of meta-analyses, performed first with one study, then with two studies, and so on, until all studies have been included in the analysis (Leimu and Koricheva 2004). It allows us to estimate the change in magnitude and direction of mean effect sizes with increasing numbers of new studies. Our datasets of resistance traits were first subdivided into studies included in the dataset of Doorduin and Vrieling (2011) and the additional ones included in our dataset, and then sorted in chronological order. Studies published in the same year were added to the analysis in random order.

We tested whether heterogeneity among effects sizes across studies $\left(Q_{\text {total }}\right)$ was significantly larger than the expected sampling error using a chi-squared test (for other examples, see van Kleunen et al. 2010 and Vilà et al. 2011). We also used chi-squared tests to assess whether mean effect sizes differed significantly among defense categories using between-group heterogeneity $\left(Q_{\mathrm{b}}\right)$ and whether the remaining within-group heterogeneity $\left(Q_{\mathrm{w}}\right)$ was significantly larger than the expected sampling error. Although there frequently is a bias against publishing negative results, funnel-plots and rank-correlation tests (Begg and Mazumdar 1994) for each defense category revealed that publication bias did not significant affect the results (Appendix S6: Table S1; Fig. S1).

\section{RESULTS}

Averaged over all studies, there was significant variability in the effect size of differences in defense between introduced and native populations $\left(Q_{\text {total }}=3257.17\right.$, df $\left.=158, P<0.0001\right)$. Mean effect sizes differed significantly among the defense categories $\left(Q_{\mathrm{b}}=40.97, \mathrm{df}=9, P<0.0001\right)$, and within each category $\left(Q_{\mathrm{w}}=2159.14, \mathrm{df}=149, \quad P<0.0001\right)$. The latter indicates that, for each defense category, the magnitude or direction of effect sizes varied significantly across studies.

\section{Physical and chemical resistance traits}

Introduced populations had lower levels of digestibility reducers than native populations, but they had similar levels of physical defenses and toxins (Table 2; Fig. 2a). Cumulative meta-analysis revealed that when studies were added to the analysis in chronological order, the cumulative effect sizes of resistance traits first increased slightly from initially negative values and then stabilized around mean values not significantly different from zero, while the confidence intervals continued to decrease (Appendix S7: Figs. S1, S2).

TABLE 2. Mean effect sizes and 95\% confidence intervals (CI) of differences in defense between introduced and native populations of invasive plants.

\begin{tabular}{|c|c|c|c|c|c|c|c|c|}
\hline \multicolumn{3}{|c|}{ Defense category } & \multirow{2}{*}{$\begin{array}{l}\text { Number of } \\
\text { effect sizes }\end{array}$} & \multirow{2}{*}{$\begin{array}{c}\text { Mean } \\
\text { effect size }\end{array}$} & \multirow{2}{*}{$\begin{array}{l}\text { Lower } \\
95 \% \text { CI }\end{array}$} & \multirow{2}{*}{$\begin{array}{l}\text { Upper } \\
95 \% \mathrm{CI}\end{array}$} & \multirow[b]{2}{*}{$Z$} & \multirow[b]{2}{*}{$P$} \\
\hline Type of defense & Herbivore & Measure & & & & & & \\
\hline \multirow[t]{3}{*}{ Resistance traits } & - & Physical defense & 14 & 0.025 & -0.143 & 0.193 & 0.294 & 0.769 \\
\hline & - & Digestibility-reducers & 9 & -0.264 & -0.493 & -0.035 & -2.263 & 0.024 \\
\hline & - & Toxin & 20 & 0.098 & -0.062 & 0.258 & 1.204 & 0.229 \\
\hline \multirow[t]{4}{*}{ Resistance effects } & $\mathrm{S}$ & Damage by herbivore & 14 & -0.254 & -0.417 & -0.092 & -3.072 & 0.002 \\
\hline & $\mathrm{S}$ & Performance of herbivore & 21 & -0.027 & -0.123 & 0.070 & -0.538 & 0.591 \\
\hline & G & Damage by herbivore & 20 & 0.324 & 0.122 & 0.526 & 3.149 & 0.002 \\
\hline & $\mathrm{G}$ & Performance of herbivore & 17 & -0.021 & -0.162 & 0.121 & -0.285 & 0.776 \\
\hline \multirow[t]{3}{*}{ Tolerance } & $\mathrm{S}$ & - & 15 & -0.016 & -0.114 & 0.081 & -0.327 & 0.744 \\
\hline & $\mathrm{G}$ & - & 16 & 0.108 & 0.007 & 0.208 & 2.092 & 0.036 \\
\hline & SH & - & 13 & -0.007 & -0.062 & 0.047 & -0.262 & 0.793 \\
\hline
\end{tabular}

Notes: Significant differences are marked in bold. Positive mean effect sizes of resistance traits, resistance effects and tolerance indicate that the introduced populations have higher levels of defenses than the native populations. S, specialist herbivores; $\mathrm{G}$, generalist herbivores; $\mathrm{SH}$, simulated herbivory. 

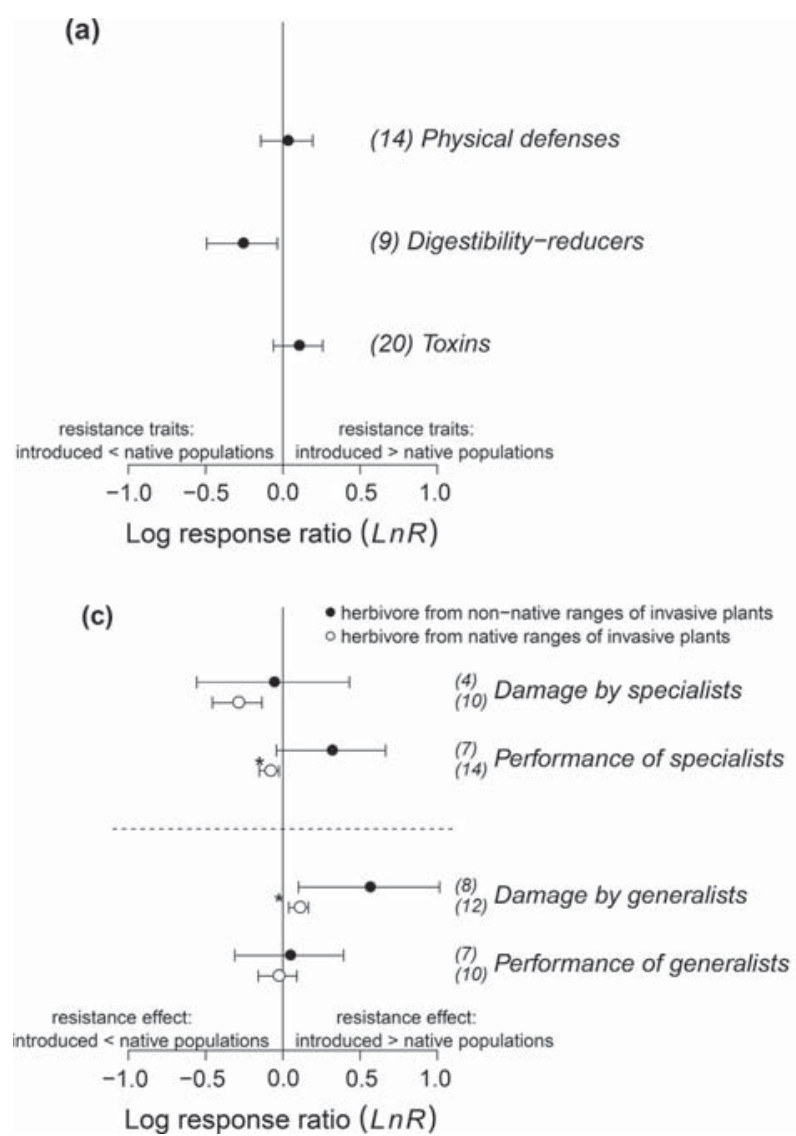
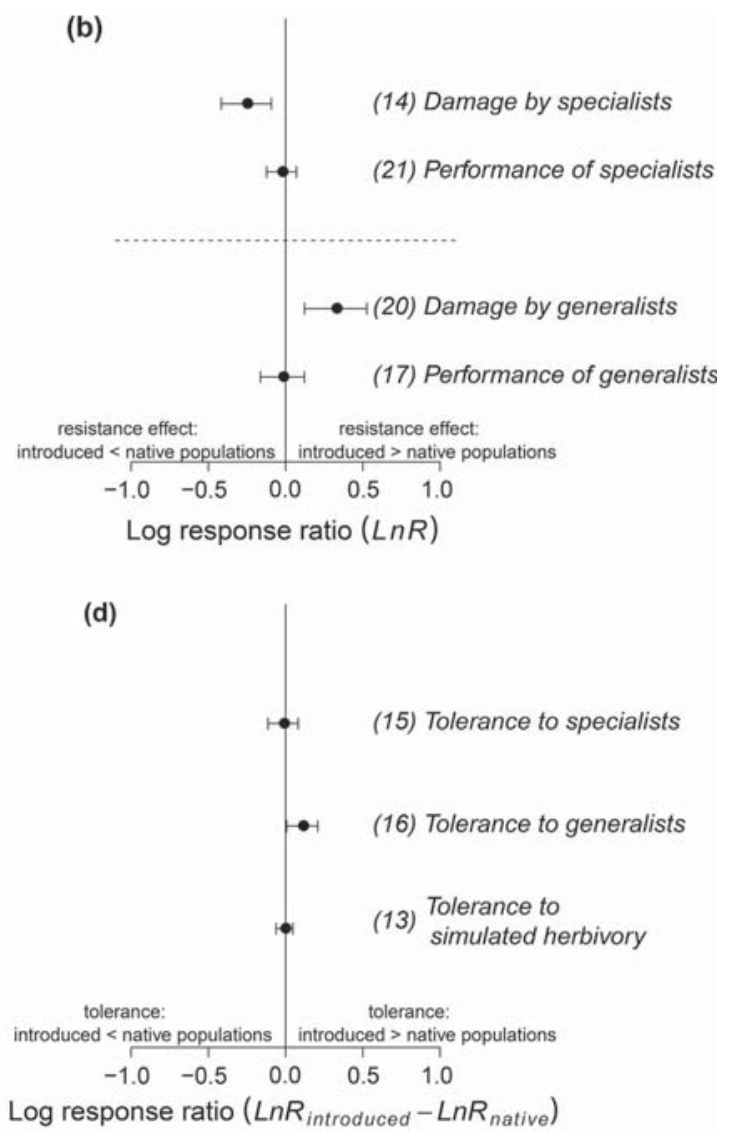

FIG. 2. Mean effect sizes (log response ratio, $\operatorname{Ln} R$ ) of differences in resistance traits (a) and resistance effects (b) between introduced and native populations of invasive plant species. (c) Effect of herbivore origin on the mean effect sizes (log response ratio, $\operatorname{Ln} R$ ) of differences in resistance effects between introduced and native populations of invasive plants. Mean effect sizes $(\log$ response ratio, $\operatorname{Ln} R$ or $\operatorname{Ln} R_{\text {difference }}$ ) of differences in tolerance (d) between introduced and native populations of invasive plant species. Positive effect sizes indicate increased defenses in introduced compared to native populations, and negative effect sizes indicate the opposite. Error bars indicate $95 \%$ confidence intervals, and a mean effect size is significantly different from zero when its $95 \%$ confidence interval does not include zero. Asterisks denote significant differences between ranges within a subgroup. Numbers in parentheses denote the numbers of studies.

\section{Resistance effects on plant damage and herbivore performance}

The analysis of resistance effects showed that, when measured in terms of damage caused by herbivores, introduced populations of invasive plants had a decreased resistance against specialist herbivores, and an increased resistance against generalist herbivores, relative to native populations (Table 2; Fig. 2b). However, the differences in resistance effects were not significant when they were measured in terms of performance of the herbivores (Table 2, Fig. 2b).

The mean effect sizes of differences in performance of specialist herbivores between introduced and native populations depended on the origin of the herbivore $\left(\chi^{2}=8.677, \mathrm{df}=1\right.$, $P=0.003)$ : whereas introduced and native populations did not differ in resistance against specialists collected from the nonnative ranges of the invasive plant species, introduced populations had a lower resistance than native populations against specialists collected from the native ranges (Appendix S4: Table S1; Fig. 2c). The origin of generalist herbivores also significantly affected the differences in damage incurred by introduced and native populations $\left(\chi^{2}=5.869, \mathrm{df}=1, P=0.015\right)$ : whereas introduced populations had, relative to native populations, an increased resistance against generalists of both origins, the magnitude of the mean effect size was larger when the generalist had been collected from the non-native range instead of the native range of the invasive plant species (Appendix S4: Table S1; Fig. 2c). Herbivore origin did not significantly affect the mean effect sizes of damage by specialists $\left(\chi^{2}=0.793\right.$, $\mathrm{df}=1, P=0.373$; Fig. 2b), or performance of generalists $\left(\chi^{2}=0.305, \mathrm{df}=1, P=0.581 ;\right.$ Fig. $\left.2 \mathrm{c}\right)$.

\section{Tolerance}

Whereas introduced and native populations did not significantly differ in tolerance to specialist herbivores and simulated herbivory, introduced populations had a higher tolerance to generalist herbivores than natives had (Table 2; Fig. 2d). Herbivore origin did not significantly affect mean effect sizes of differences in tolerance between introduced and native populations to the three categories of herbivory treatments (Appendix S4: Table S1; Fig. S1).

\section{DisCUSSION}

It is under debate whether specialist and generalist herbivores interact with plants in distinct ways (Ali and Agrawal 2012) and have contrasting effects on defense evolution in invasive plants (Blossey and Nötzold 1995, Müller-Schärer 
et al. 2004). Our meta-analysis provides strong evidence in support of the Shifting Defense hypothesis in evolution of resistance against specialist and generalist herbivores. In contrast, we found no consistent evidence for evolution of tolerance, except for increased tolerance to generalists in introduced populations of invasive plants. Overall, our results bolster the idea that specialist and generalist herbivores play different roles in successful invasion of alien plants (Keane and Crawley 2002, Müller-Schärer et al. 2004, Joshi and Vrieling 2005, Parker et al. 2006).

\section{Evolution of resistance against specialist and generalist herbivores}

A major finding of our study is that, measured in terms of damage by herbivores, invasive plants generally showed decreased resistance against specialist herbivores and increased resistance against generalist herbivores following introduction. This result strongly supports the Shifting Defense hypothesis. As recently proposed by several studies (Orians and Ward 2010, Schultheis et al. 2015, Gruntman et al. 2017), an evolutionary shift in resistance could help alien plants to become invasive when it helps them overcome biotic resistance from newly encountered generalists. In apparent contrast to our finding, another recent meta-analysis by Felker-Quinn et al. (2013) found that plant resistance against generalists and specialists did not differ between introduced and native populations. Felker-Quinn et al. (2013), however, only looked at resistance effects in terms of performance of the herbivores, which also did not differ between introduced and native populations in our study.

The different results we found for the two measures of resistance effects, damage by and performance of herbivores, might arise from at least two aspects. Firstly, from the perspective of the plant, reducing damage by herbivores might provide a more immediate advantage than decreasing performance of the herbivore. Secondly, decreased performance of the herbivore (e.g., longer development time) may sometimes lead to extended feeding (Karban 2011), and would only have a long-term benefit to the plant (or its offspring) when population growth of the herbivore is reduced. Whatever the exact reason for why the results for damage by herbivores and performance of herbivores differ, our metaanalysis suggests that changes in both measures of resistance effects do not necessarily coincide.

Our meta-analysis showed that introduced and native populations had similar levels of physical defenses and toxins. In contrast to our study, the meta-analysis of Doorduin and Vrieling (2011) found higher levels of toxins in introduced than in native populations. Cumulative meta-analysis revealed that our study, with additional data, had a smaller confidence interval for the mean effect sizes of toxins (Appendix S7: Fig. S2), and thus provides a more powerful dataset than that of Doorduin and Vrieling (2011). We offer two explanations for the inconsistent evidence for evolution of physical defenses and toxins. Firstly, toxins and physical defenses have been reported to be effective against both specialist and generalist herbivores. For example, the furanocoumarins, which are toxins in Pastinaca sativa, are known to provide resistance against a specialist herbivore (Berenbaum et al. 1986). Consequently, opposing selective pressures due to release from specialist enemies and biotic resistance by generalists on these resistance traits could counter each other. Secondly, resistance traits have pleiotropic effects, such as protection against UV radiation and in allelopathic interactions with competitors (Zheng et al. 2015), and therefore could be selected by environmental changes other than herbivory (e.g., competition).

We found that introduced populations had lower levels of digestibility-reducers than native populations, and that correspondingly there was a decreased resistance effect on specialist herbivores in introduced populations. This supports the idea that digestibility-reducers provide resistance against specialist herbivores (Müller-Schärer et al. 2004). The reduction in costly digestibility-reducers in introduced populations could make additional resources available for growth and thereby increase competitive ability, as predicted by the Evolution of Increased Competitive Ability (EICA) hypothesis (Blossey and Nötzold 1995).

The differences between introduced and native populations in digestibility-reducers and corresponding resistance effects on specialist herbivores, as well as in resistance effects on generalist herbivores are in accord with the Shifting Defense hypothesis. In contrast, toxins and physical defenses showed no consistent differences between native and introduced populations, perhaps because of the variable selective pressures on such defenses. The incomplete consistency between resistance traits and resistance effects may also indicate that the assumption that toxins mainly resist generalists and physical defenses mainly resist specialists is wrong. We recommend that future studies integrate diverse resistance traits with resistance effects, and also consider pleiotropic effects (e.g., allelopathy) of resistance traits simultaneously. In addition, preference of herbivores, another important measure of resistance effect, has been largely ignored and deserves further investigation.

\section{Herbivore origin matters}

Another important finding of our meta-analysis is that herbivore origin significantly affected the resistance difference between introduced and native populations. We found that introduced and native populations did not differ in resistance-measured as performance of the herbivoreagainst specialist herbivores collected from the non-native ranges of the invasive plant species, whereas introduced populations had decreased resistance against specialists collected from the native ranges. This result suggests that invasive plants might not necessarily fall behind in the coevolutionary arms race with their specialist herbivores. Introduced populations might have lost adaptation to the specialists collected from their native range, which no longer co-occur with them. However, they are adapted to some extent to the specialists collected from the non-native range, which currently co-occur with them. A possible artifact is that biological control agents, which were specifically selected by humans, were frequently (8 out of 11 studies) used when measuring resistance effects against specialists collected from the nonnative ranges. However, our study revealed that whether biological control agents were used or not did not significantly affect the result for the evolution of resistance against specialists (Appendix S4: Table S1), and hence our results can be considered robust. 
We found that the magnitude of evolution of increased resistance - measured as damage by generalist herbivoreswas larger when the generalists had been collected from the non-native range of the invasive plant species than when they had been collected from the native range. This suggests that the evolutionary change of resistance in invasive plants is more specific to deter generalists from the non-native ranges of the plant. This reinforces the idea that biotic resistance from generalists in non-native ranges might have imposed strong selection on invasive plants (Joshi and Vrieling 2005, Gruntman et al. 2017). Interestingly, introduced populations had increased resistance against generalists from the native and non-native ranges, despite the fact that the former did not co-occur with the introduced populations. This might suggest that generalists from the non-native and native ranges of invasive plants are somewhat similar, so that increased resistance evolved in introduced populations is effective against both types of generalists.

\section{Evolution of tolerance in invasive plants}

Despite large agreement among studies in how resistance changes following introduction of invasive plants, we found no consistent patterns of evolution in tolerance to specialist and simulated herbivory. However, introduced populations had a higher tolerance to generalist herbivores than native populations had. This finding, along with increased resistance against generalists in introduced populations, does not support the classical theory that there is a tradeoff between resistance and tolerance (van der Meijden et al. 1988). Recent theoretical studies showed that when herbivore pressure is high, as is the case for introduced populations that are released from specialists but encounter high biotic resistance from generalists, a mixture of both resistance and tolerance is possible (Fornoni et al. 2004, Núñez-Farfán et al. 2007).

It must be kept in mind that the test for tolerance evolution in invasive plants might be affected by the method used to measure tolerance (Tiffin and Inouye 2000, Lehtilä 2003). Given that introduced and native populations differ in resistance against herbivores (i.e., were damaged to different degrees), it might be useful to do studies that compare tolerance between introduced and native populations using a gradient of damage levels. However, the few studies that did this did not find effects of herbivore-damage levels on the tolerance difference between introduced and native populations (Bossdorf et al. 2004, Gard et al. 2013). Interestingly, Triadica sebifera - the only invasive woody species for which evolution of tolerance has been studied so far - appears to have increased tolerance following introduction (Appendix S4: Table S1; Fig. S2c). This might suggest that life form could affect evolution of tolerance, and calls for more studies on woody species.

\section{Conclusions}

Our meta-analysis shows for the first time that origin of the herbivore used in a study could be an important source of variation in the results on evolution of resistance against herbivores in invasive plants. Most importantly, we integrated resistance effects, resistance traits and tolerance into a comprehensive framework, and show with the largest dataset available up to date that resistance of invasive plants, when measured as damage by herbivores, has evolved after introduction into non-native ranges. These results are in remarkable agreement with the predictions of the Shifting Defense hypothesis (Müller-Schärer et al. 2004, Joshi and Vrieling 2005). Our study indicates that two major mechanisms -release from specialist enemies and biotic resistance by generalist herbivores- may be pivotal in the evolution of defenses against herbivores in invasive plants.

\section{ACKNOWLedgMents}

We thank S. Wei and Y. Liu for technical advice with regard to meta-analysis, editor and two anonymous reviewers for constructive comments to improve earlier drafts of this manuscript. We apologize to all those authors whose work we might have missed. This study was financially supported by National Key Research and Development Program (Grant No. 2016YFC1200600) and National Science Foundation of China (Grant No. 31070369, 31370433, 41630528).

\section{Literature Cited}

Ali, J. G., and A. A. Agrawal. 2012. Specialist versus generalist insect herbivores and plant defense. Trends in Plant Science 17:293-302.

Begg, C. B., and M. Mazumdar. 1994. Operating characteristics of a rank correlation test for publication bias. Biometrics 50: 1088-1101.

Berenbaum, M. R., A. R. Zangerl, and J. K. Nitao. 1986. Constraints on chemical coevolution: wild parsnips and the parsnip webworm. Evolution 40:1215-1228.

Blossey, B., and R. Nötzold. 1995. Evolution of increased competitive ability in invasive nonindigenous plants - a hypothesis. Journal of Ecology 83:887-889.

Borenstein, M., L. V. Hedges, J. P. T. Higgins, and H. R. Rothstein. 2009. Introduction to meta-analysis. A John Wiley and Sons, West Sussex, UK.

Bossdorf, O., H. Auge, L. Lafuma, W. E. Rogers, E. Siemann, and D. Prati. 2005. Phenotypic and genetic differentiation between native and introduced plant populations. Oecologia 144:1-11.

Bossdorf, O., S. Schroder, D. Prati, and H. Auge. 2004. Palatability and tolerance to simulated herbivory in native and introduced populations of Alliaria petiolata (Brassicaceae). American Journal of Botany 91:856-862.

Bukovinszky, T., R. Gols, A. A. Agrawal, C. Roge, T. M. Bezemer, A. Biere, and J. A. Harvey. 2014. Reciprocal interactions between native and introduced populations of common milkweed, Asclepias syriaca, and the specialist aphid, Aphis nerii. Basic and Applied Ecology 15:444-452.

Carmona, D., and J. Fornoni. 2013. Herbivores can select for mixed defensive strategies in plants. New Phytologist 197:576-585.

Cates, R. G., and D. F. Rhoades. 1977. Pratterns in production of anti-herbiovre chemical defenses in plant communities. Biochemical Systematics and Ecology 5:185-193.

Colautti, R. I., and S. C. H. Barrett. 2013. Rapid adaptation to climate facilitates range expansion of an invasive plant. Science 342:364-366.

Colautti, R. I., J. L. Maron, and S. C. H. Barrett. 2009. Common garden comparisons of native and introduced plant populations: latitudinal clines can obscure evolutionary inferences. Evolutionary Applications 2:187-199.

Coley, P. D., J. P. Bryant, and F. S. Chapin. 1985. Resource availability and plant antiherbivore defense. Science 230:895-899.

de Jong, T. J., and T. Lin. 2017. How to quantify plant tolerance to loss of biomass? Ecology and Evolution 7:3080-3086.

Doorduin, L. J., and K. Vrieling. 2011. A review of the phytochemical support for the shifting defence hypothesis. Phytochemistry Reviews 10:99-106.

Elton, C. S. 1958. The ecology of invasion by animals and plants. Methuen, London, UK. 
Feeny, P. 1975. Biochemical coevolution between plants and their insect herbivores. Pages 3-19 in L. Gilbert and P. Raven, editors. Coevolution of animals and plants. University of Texas Press, Austin, Texas, USA.

Feeny, P. 1976. Plant Apparency and Chemical Defense. Pages 1-40 in J. W. Wallace and R. L. Mansell, editors. Biochemical interaction between plants and insects. Springer US, Boston, Massachusetts, USA.

Felker-Quinn, E., J. A. Schweitzer, and J. K. Bailey. 2013. Meta-analysis reveals evolution in invasive plant species but little support for Evolution of Increased Competitive Ability (EICA). Ecology and Evolution 3:739-751.

Fornoni, J., J. Nunez-Farfan, P. L. Valverde, and M. D. Rausher. 2004. Evolution of mixed strategies of plant defense allocation against natural enemies. Evolution 58:1685-1695.

Gard, B., F. Bretagnolle, F. Dessaint, and B. Laitung. 2013. Invasive and native populations of common ragweed exhibit strong tolerance to foliar damage. Basic and Applied Ecology 14:28-35.

Gruntman, M., U. Segev, G. Glauser, and K. Tielbörger. 2017. Evolution of plant defences along an invasion chronosequence: defence is lost due to enemy release - but not forever. Journal of Ecology 105:255-264.

Huang, W., E. Siemann, G. S. Wheeler, J. Zou, J. Carrillo, and J. Ding. 2010. Resource allocation to defence and growth are driven by different responses to generalist and specialist herbivory in an invasive plant. Journal of Ecology 98:1157-1167.

Joshi, J., and K. Vrieling. 2005. The enemy release and EICA hypothesis revisited: incorporating the fundamental difference between specialist and generalist herbivores. Ecology Letters 8:704-714.

Karban, R. 2011. The ecology and evolution of induced resistance against herbivores. Functional Ecology 25:339-347.

Keane, R. M., and M. J. Crawley. 2002. Exotic plant invasions and the enemy release hypothesis. Trends in Ecology \& Evolution 17:164-170.

Kempel, A., M. Schadler, T. Chrobock, M. Fischer, and M. van Kleunen. 2011. Tradeoffs associated with constitutive and induced plant resistance against herbivory. Proceedings of the National Academy of Sciences of the United States of America 108:5685-5689.

Koricheva, J. 2002. Meta-analysis of sources of variation in fitness costs of plant antiherbivore defenses. Ecology 83:176-190.

Lajeunesse, M. J. 2015. Bias and correction for the log response ratio in ecological meta-analysis. Ecology 96:2056-2063.

Lee, C. E. 2002. Evolutionary genetics of invasive species. Trends in Ecology \& Evolution 17:386-391.

Leger, E. A., and M. L. Forister. 2005. Increased resistance to generalist herbivores in invasive populations of the California poppy (Eschscholzia californica). Diversity and Distributions 11:311-317.

Lehtilä, K. 2003. Precision of herbivore tolerance experiments with imposed and natural damage. Evolution 57:677-680.

Leimu, R., and J. Koricheva. 2004. Cumulative meta-analysis: a new tool for detection of temporal trends and publication bias in ecology. Proceedings of the Royal Society of London. Series B: Biological Sciences 271:1961-1966.

Leimu, R., and J. Koricheva. 2006. A meta-analysis of tradeoffs between plant tolerance and resistance to herbivores: combining the evidence from ecological and agricultural studies. Oikos 112:1-9.

Levine, J. M., P. B. Adler, and S. G. Yelenik. 2004. A meta-analysis of biotic resistance to exotic plant invasions. Ecology Letters 7:975-989.

Liu, H., and P. Stiling. 2006. Testing the enemy release hypothesis: a review and meta-analysis. Biological Invasions 8:1535-1545.

Liu, M., F. Zhou, X. Pan, Z. Zhang, M. B. Traw, and B. Li. 2017. Specificity of herbivore-induced responses in an invasive species, Alternanthera philoxeroides (alligator weed). Ecology and Evolution 8:59-70.

Maron, J. L., and M. Vilà. 2001. When do herbivores affect plant invasion? Evidence for the natural enemies and biotic resistance hypotheses. Oikos 95:361-373.
Mesa, J. M., D. R. Scholes, J. A. Juvik, and K. N. Paige. 2017. Molecular constraints on resistance-tolerance trade-offs. Ecology 98:2528-2537.

Meyer, G. A., and H. M. Hull-Sanders. 2008. Altered patterns of growth, physiology and reproduction in invasive genotypes of Solidago gigantea (Asteraceae). Biological Invasions 10:303-317.

Morrison, W. E., and M. E. Hay. 2011. Herbivore preference for native vs. exotic plants: generalist herbivores from multiple continents prefer exotic plants that are evolutionarily naïve. PLoS ONE 6:e17227.

Müller-Schärer, H., U. Schaffner, and T. Steinger. 2004. Evolution in invasive plants: implications for biological control. Trends in Ecology \& Evolution 19:417-422.

Núñez-Farfán, J., J. Fornoni, and P. L. Valverde. 2007. The evolution of resistance and tolerance to herbivores. Annual Review of Ecology Evolution and Systematics 38:541-566.

Oduor, A. M. O., R. Leimu, and M. van Kleunen. 2016. Invasive plant species are locally adapted just as frequently and at least as strongly as native plant species. Journal of Ecology 104: 957-968.

Orians, C. M., and D. Ward. 2010. Evolution of plant defenses in nonindigenous environments. Annual Review of Entomology 55:439-459.

Pan, X., X. Jia, J. Chen, and B. Li. 2012. For or against: the importance of variation in growth rate for testing the EICA hypothesis. Biological Invasions 14:1-8.

Parker, J. D., D. E. Burkepile, and M. E. Hay. 2006. Opposing effects of native and exotic herbivores on plant invasions. Science 311:1459-1461.

R Development Core Team. 2013. R: a language and environment for statistical computing. R Foundation for Statistical Computing, Vienna, Austria. http://www.R-project.org/

Ridenour, W. M., J. M. Vivanco, Y. Feng, J.-I. Horiuchi, and R. M. Callaway. 2008. No evidence for trade-offs: Centaurea plants from America are better competitors and defenders. Ecological Monographs 78:369-386.

Schaffner, U., W. M. Ridenour, V. C. Wolf, T. Bassett, C. Müller, H. Müller-Schärer, S. Sutherland, C. J. Lortie, and R. M. Callaway. 2011. Plant invasions, generalist herbivores, and novel defense weapons. Ecology 92:829-835.

Schultheis, E. H., A. E. Berardi, and J. A. Lau. 2015. No release for the wicked: enemy release is dynamic and not associated with invasiveness. Ecology 96:2446-2457.

Schultz, J. C. 1989. Tannin-Insect Interactions. Pages 417-433 in R. W. Hemingway, J. J. Karchesy, and S. J. Branham, editors. Chemistry and significance of condensed tannins. Springer US, Boston, Massachusetts, USA.

Seastedt, T. R. 2015. Biological control of invasive plant species: a reassessment for the Anthropocene. New Phytologist 205:490-502.

Seebens, H., et al. 2017. No saturation in the accumulation of alien species worldwide. Nature Communications 8:14435.

Smilanich, A. M., R. M. Fincher, and L. A. Dyer. 2016. Does plant apparency matter? Thirty years of data provide limited support but reveal clear patterns of the effects of plant chemistry on herbivores. New Phytologist 210:1044-1057.

Sorte, C. J., et al. 2013. Poised to prosper? A cross-system comparison of climate change effects on native and non-native species performance. Ecology Letters 16:261-270.

Stamp, N. 2003. Out of the quagmire of plant defense hypotheses. Quarterly Review of Biology 78:23-55.

Stowe, K. A., R. J. Marquis, C. G. Hochwender, and E. L. Simms. 2000. The evolutionary ecology of tolerance to consumer damage. Annual Review of Ecology and Systematics 31:565-595.

Tiffin, P., and B. D. Inouye. 2000. Measuring tolerance to herbivory: accuracy and precision of estimates made using natural versus imposed damage. Evolution 54:1024-1029.

van der Meijden, E. 1996. Plant defence, an evolutionary dilemma: contrasting effects of (specialist and generalist) herbivores and natural enemies. Entomologia Experimentalis Et Applicata 80: 307-310. 
van der Meijden, E., M. Wijn, and H. J. Verkaar. 1988. Defense and regrowth, alternative plant strategies in the struggle against herbivores. Oikos 51:355-363.

van Kleunen, M., and B. Schmid. 2003. No evidence for an evolutionary increased competitive ability in an invasive plant. Ecology 84:2816-2823.

van Kleunen, M., E. Weber, and M. Fischer. 2010. A meta-analysis of trait differences between invasive and non-invasive plant species. Ecology Letters 13:235-245.

van Kleunen, M., et al. 2015. Global exchange and accumulation of non-native plants. Nature 525:100-103.

Viechtbauer, W. 2010. Conducting meta-analyses in $\mathrm{R}$ with the metafor package. Journal of Statistical Software 36:48

Vilà, M., J. L. Espinar, M. Hejda, P. E. Hulme, V. Jarosik, J. L. Maron,

J. Pergl, U. Schaffner, Y. Sun, and P. Pysek. 2011. Ecological impacts of invasive alien plants: a meta-analysis of their effects on species, communities and ecosystems. Ecology Letters 14: 702-708.

Wolfe, L. M., J. A. Elzinga, and A. Biere. 2004. Increased susceptibility to enemies following introduction in the invasive plant Silene latifolia. Ecology Letters 7:813-820.

Zhang, Z. J., X. Y. Pan, Z. Y. Zhang, K. S. He, and B. Li. 2015. Specialist insect herbivore and light availability do not interact in the evolution of an invasive plant. PLoS ONE 10: e0139234.

Zheng, Y. L., Y. L. Feng, L. K. Zhang, R. M. Callaway, A. ValienteBanuet, D. Q. Luo, Z. Y. Liao, Y. B. Lei, G. F. Barclay, and C. Silva-Pereyra. 2015. Integrating novel chemical weapons and evolutionarily increased competitive ability in success of a tropical invader. New Phytologist 205:1350-1359. 